\title{
Ultrahigh-speed distributed Brillouin reflectometry
}

\author{
Yosuke Mizuno $^{1}$, Neisei Hayashi ${ }^{2}$, Hideyuki Fukuda ${ }^{3}$, Kwang Yong Song ${ }^{4}$ and Kentaro Nakamura ${ }^{1}$
}

Optical fibre sensors based on Brillouin scattering have been vigorously studied in the context of structural health monitoring on account of their capacity for distributed strain and temperature measurements. However, real-time distributed strain measurement has been achieved only for two-end-access systems; such systems reduce the degree of freedom in embedding the sensors into structures, and furthermore render the measurement no longer feasible when extremely high loss or breakage occurs at a point along the sensing fibre. Here, we demonstrate real-time distributed measurement with an intrinsically one-end-access reflectometry configuration by using a correlation-domain technique. In this method, the Brillouin gain spectrum is obtained at high speed using a voltage-controlled oscillator, and the Brillouin frequency shift is converted into a phase delay of a synchronous sinusoidal waveform; the phase delay is subsequently converted into a voltage, which can be directly measured. When a single-point measurement is performed at an arbitrary position, a strain sampling rate of up to $100 \mathrm{kHz}$ is experimentally verified by detecting locally applied dynamic strain at $1 \mathrm{kHz}$. When distributed measurements are performed at 100 points with 10 times averaging, a repetition rate of $100 \mathrm{~Hz}$ is verified by tracking a mechanical wave propagating along the fibre. Some drawbacks of this ultrahigh-speed configuration, including the reduced measurement accuracy, lowered spatial resolution and limited strain dynamic range, are also discussed.

Light: Science \& Applications (2016) 5, e16184; doi:10.1038/lsa.2016.184; published online 16 December 2016

Keywords: Brillouin scattering; distributed strain and temperature sensing; high-speed measurement; nonlinear optics; optical fibre sensors

\section{INTRODUCTION}

Brillouin scattering ${ }^{1}$ is regarded as one of the most promising principles of fibre-optic sensing in terms of the feasibility it affords for measurements of strain and temperature distribution. The Brillouin-based distributed sensors developed so far can be classified into two types: 'analysis' in which two light beams are injected into both ends of a fibre under test (FUT) and 'reflectometry' in which a light beam is injected into only one end of an FUT. The former category includes Brillouin optical time-domain analysis (BOTDA $)^{2-12}$, Brillouin optical frequencydomain analysis (BOFDA) $)^{13-16}$ and Brillouin optical correlationdomain analysis (BOCDA $)^{12,17-25}$, whereas the latter category includes Brillouin optical time-domain reflectometry (BOTDR) $)^{26-30}$ and Brillouin optical correlation-domain reflectometry (BOCDR $)^{31-37}$. For each technique, a number of different configurations have been proposed in order to improve the sensing performance in terms of spatial resolution, measurement range, signal-to-noise ratio (SNR), sampling rate, repetition rate, strain and temperature sensitivity, strain dynamic range, system simplicity and cost efficiency. Here, we focus on Brillouin sensors with a high sampling (or repetition) rate for distributed dynamic strain measurement.

To date, extremely high sampling rates have been achieved in BOTDA $^{3-11}$ and BOCDA ${ }^{19,20,25}$. In particular, Voskoboinik et al. ${ }^{3}$ have proposed the idea of using multiple pumps and probes in BOTDA to avoid time-consuming frequency sweeping, thus leading to a potential increase in measurement speed at the expense of frequency granularity. Furthermore, inspired by the idea of Bernini et al. ${ }^{4}$, Peled et al. ${ }^{5}$ have developed slope-assisted BOTDA, which interrogates the FUT with a single frequency located at the middle of the slope of the local Brillouin gain spectrum (BGS), thereby enabling a single pump pulse to sample the strain distribution along the full length of the FUT. By synthesising an adaptable probe wave, an FUT with an arbitrary Brillouin frequency shift (BFS) distribution can be interrogated, though the strain dynamic range is limited by the size of the linear section of the BGS. Peled et al. ${ }^{6}$ have also demonstrated dynamic BOTDA measurements by implementing the fast switching of optical frequency using high-performance arbitrary-waveform generators, though the measurement time becomes longer as the length of the FUT increases. In addition, Taki et al. ${ }^{7}$ have proposed the use of cyclic pulse coding based on quasi-periodic bit sequences in BOTDA for an FUT with a length on the order of kilometres. This configuration allows for real-time decoding, resulting in a substantial reduction in the number of averages required to guarantee an acceptable SNR. Performance improvements for these distributed dynamic sensors based on BOTDA are still being aggressively studied ${ }^{8-11}$.

As for BOCDA, Song et al. ${ }^{19}$ have achieved a $1 \mathrm{kHz}$ sampling rate at a single location using a time-division pump-probe generation scheme

${ }^{1}$ Institute of Innovative Research, Tokyo Institute of Technology, Midori-ku, Yokohama 226-8503, Japan; ${ }^{2}$ Research Center for Advanced Science and Technology, The University of Tokyo, Meguro-ku, Tokyo 153-8904, Japan; ${ }^{3}$ Servo Laboratory, FANUC Corporation, Oshino-mura, Yamanashi 401-0597, Japan and ${ }^{4}$ Department of Physics, Chung-Ang University, Dongjak-gu, Seoul 06974, Korea

Correspondence: Y Mizuno, Email: ymizuno@sonic.pi.titech.ac.jp

Received 3 March 2016; revised 27 June 2016; accepted 29 June 2016; accepted article preview online 30 June 2016 
with optimised temporal gates and an unbalanced Mach-Zehnder delay line. By using differential frequency modulation, they have additionally demonstrated a distributed measurement over a $100-\mathrm{m}$ long FUT at a 20-Hz repetition rate with a spatial resolution of $80 \mathrm{~cm}^{20}$. Random accessibility with a $5-\mathrm{kHz}$ sampling rate has also been demonstrated by the employment of a high-speed lock-in amplifier ${ }^{25}$. In this manner, only analysis systems based not on spontaneous but on stimulated Brillouin scattering (SBS) have been exploited to acquire Brillouin signals on a real-time basis with sub-metre spatial resolution.

In general, SBS is induced by a so-called pump-probe configuration, which poses one major problem. This configuration requires the pump and probe lights to be injected into both ends of the FUT, which is not convenient for practically embedding the sensors into materials and structures; furthermore, the measurement can no longer be performed when extremely high loss or breakage occurs at a point along the FUT. Some quasi-one-end-access configurations ${ }^{16,21,22}$ have been developed by exploiting the Fresnel reflection at the open end of an FUT (cut perpendicularly to the fibre axis for high reflection, with a mirror or a small fibre loop sometimes being equipped), but they cannot perfectly overcome the measurement difficulties associated with a breakage point.

Against this background, it is of paramount importance to develop distributed Brillouin sensing technology that simultaneously provides a high sampling/repetition rate, intrinsically one-end-access interrogation, and high spatial resolution. BOTDR does not seem to be suitable because the spontaneous-Brillouin-scattered signal generated by incident optical pulses is so weak that the signal needs to be integrated numerous times ${ }^{26-30}$.

In this work, we describe a new configuration for BOCDR that satisfies these three requirements simultaneously. BOCDR is known to be an intrinsically one-end-access sensing technique with high-spatial resolution, but its highest sampling rate reported so far was lower than $20 \mathrm{~Hz}$. Here, using a voltage-controlled oscillator (VCO), the frequency sweeping for acquiring the BGS is performed at high speed without using the inherent sweeping function of an electrical spectrum analyser (ESA), which is used only to detect the signal power at a fixed frequency component. The BGS is then approximated by a sinusoidal waveform, and the BFS is converted into its phase delay, which is further converted into a voltage for direct measurement. When a single-point measurement is performed at an arbitrary position, a maximal strain sampling rate of $100 \mathrm{kHz}$ is experimentally verified by detecting dynamic strain at $1 \mathrm{kHz}$. When distributed measurements are performed at 100 points with 10 times averaging, a repetition rate of $100 \mathrm{~Hz}$ (corresponding to the maximal sampling rate $(100 \mathrm{kHz})$ divided by the number of points and the number of averages) is then proved to be feasible by tracking a propagating mechanical wave. This ultrahigh-speed configuration functions at the sacrifice of the deteriorated measurement accuracy, lowered spatial resolution, and limited range of measurable strain, which are fully discussed.

\section{MATERIALS AND METHODS}

$\mathrm{BOCDR}^{31,32}$ is known as a distributed sensing technique with intrinsic one-end accessibility and high spatial resolution. Its operating principle is based on the correlation control of continuous lightwaves in a self-heterodyne scheme. In other words, the pump light and the reference light (instead of the probe light used in BOCDA) are sinusoidally frequency-modulated at $f_{\mathrm{m}}$, producing periodical 'correlation peaks ${ }^{38}$ in the FUT. The measurement range $d_{\mathrm{m}}$, determined by the interval between the correlation peaks, is inversely proportional to $f_{\mathrm{m}}$, as follows ${ }^{32}$ :

$$
d_{\mathrm{m}}=\frac{c}{2 n f_{\mathrm{m}}}
$$

where $c$ is the velocity of light in a vacuum and $n$ is the refractive index of the fibre core. By sweeping $f_{\mathrm{m}}$, the correlation peak, i.e. the sensing position, can be scanned along the fibre to acquire a BGS or BFS distribution. The spatial resolution $\Delta z$ is given by $^{32}$ :

$$
\Delta z=\frac{c \Delta v_{\mathrm{B}}}{2 \pi n f_{\mathrm{m}} \Delta f}
$$

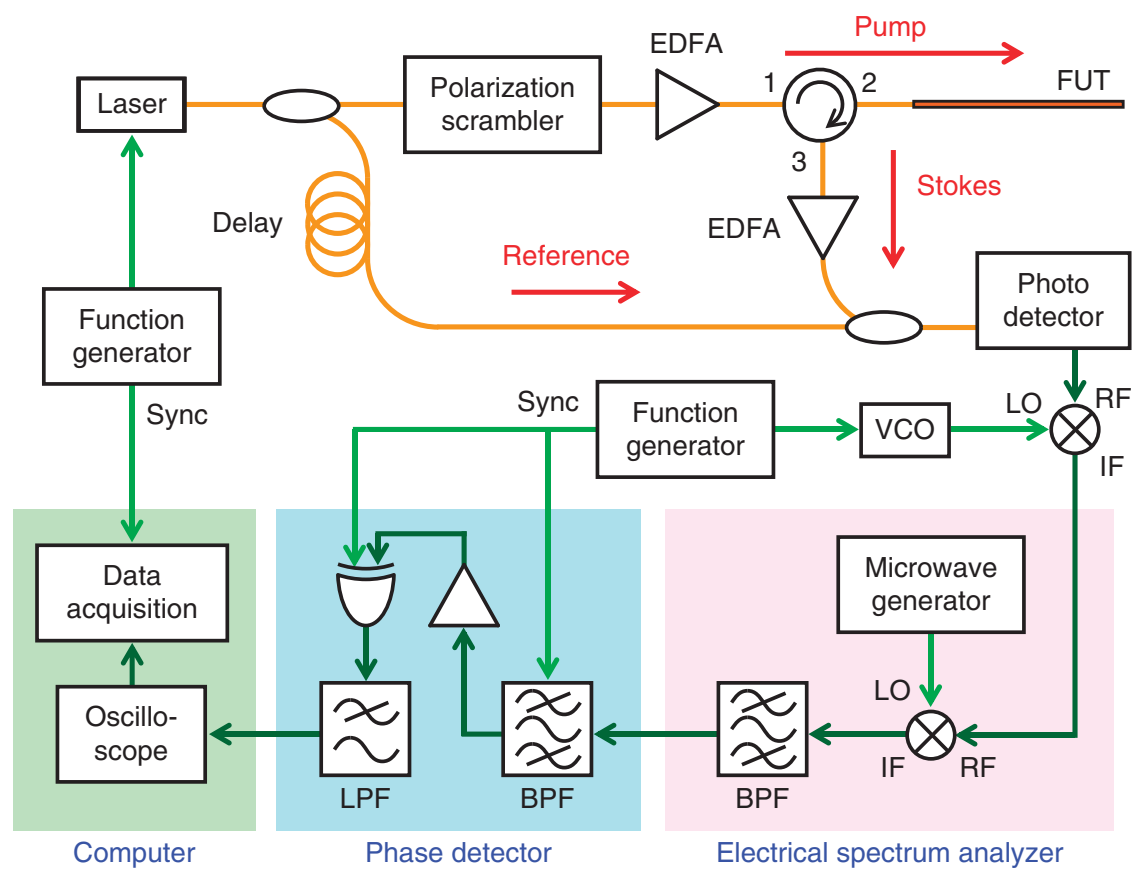

Figure 1 Configuration of ultrahigh-speed BOCDR. Experimental setup. EDFA, erbium-doped fibre amplifier; IF, intermediate frequency; LO, local oscillator; $\mathrm{RF}$, radio frequency. 
where $\Delta \nu_{\mathrm{B}}$ is the Brillouin bandwidth $(\sim 30 \mathrm{MHz}$ in silica single-mode fibres (SMFs)) and $\Delta f$ is the modulation amplitude of the optical frequency. Note that $\Delta f$ is practically limited to one half of the BFS of the FUT because of the noise caused by the Rayleigh scattering ${ }^{31,32}$. To date, the highest sampling rate including data acquisition to the computer was $19 \mathrm{~Hz}^{33}$, and was limited by the sweeping speed of an ESA.

The experimental setup of the newly configured BOCDR is schematically shown in Figure 1 . The fibre-optic parts were similar to those in a conventional $\operatorname{setup}^{35}$; a polarisation scrambler was employed in the pump path (see Supplementary Information for details). The heterodyned Brillouin signal was converted into an electrical signal using a photo detector (PD). The key point of highspeed BGS acquisition is the conversion of the BGS from the frequency domain to the time domain (Figure 2a). The PD output was mixed with the output of a VCO, the frequency of which was repeatedly swept over a range of several hundred megahertz using a function generator, resulting in the BGS scanned in the frequency domain. The power at a fixed frequency component (carefully chosen) of the mixed signal was then output from the ESA using a so-called zero-span mode, which repeatedly provided the BGS in the time domain. The BGS acquisition (determined by the repetition rate of the VCO) must be slower than the calculation time required for the BFS derivation from the BGS, and therefore we developed a method for simultaneously deriving the BFS and the BGS. The basic concept of the subsequent signal processing is shown in Figure 2b. The ESA output, which can be regarded as the BGS in the time domain, was approximated by a one-period sinusoidal waveform using a bandpass filter $(\mathrm{BPF})$ with the same central frequency as the repetition rate of the VCO. At this stage, the BFS was in one-to-one correspondence with the phase delay of the sinusoidal waveform (at the expense of the limited range of measurable strain; only a phase delay smaller than $180^{\circ}$ can be properly detected). The phase delay was then converted into a quasi-DC voltage using an electrical amplifier (AMP), an exclusive-OR (XOR) logic gate, and a low-pass filter (LPF), and was finally input to the computer via a sound board and monitored using a

a
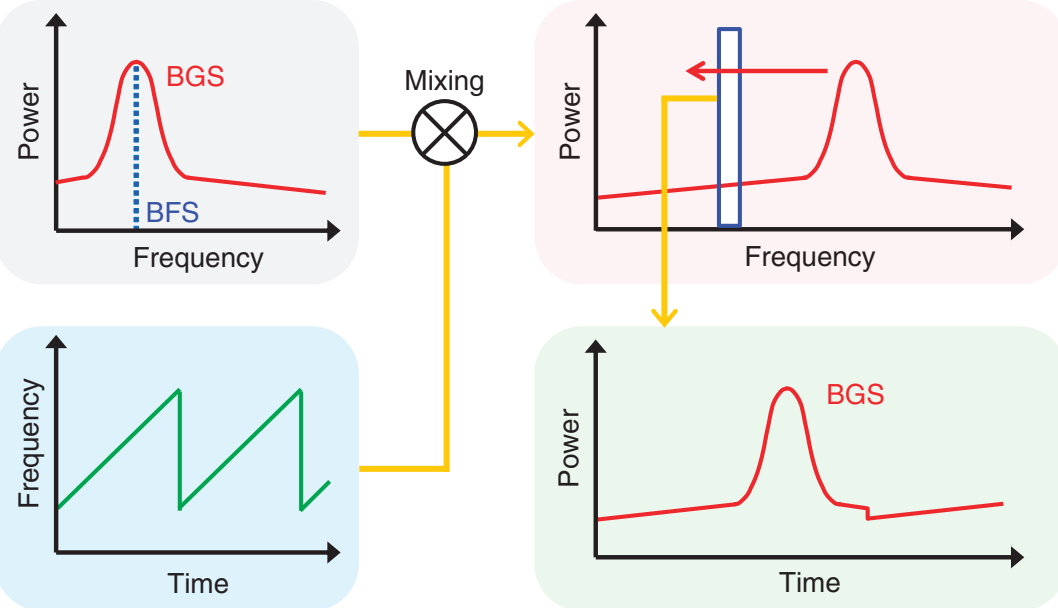

b

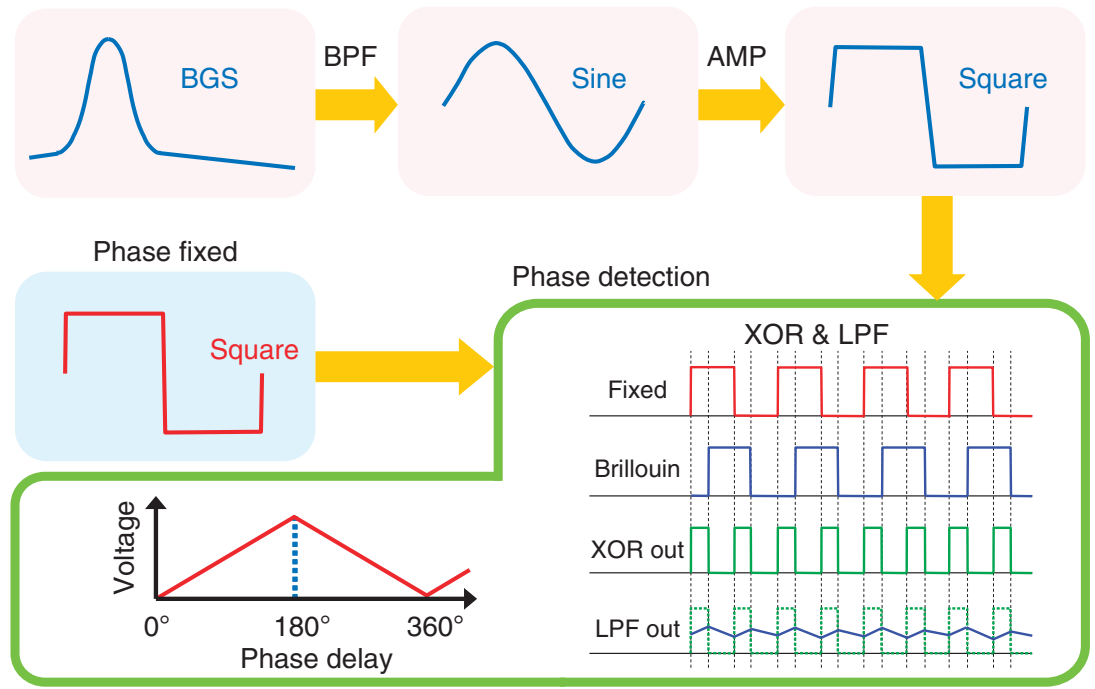

Figure 2 Principles of high-speed acquisition of BGS and BFS. (a) BGS acquisition. By mixing with a frequency-swept microwave, the BGS originally observed in the frequency domain can be obtained in the time domain at high speed. (b) BFS acquisition. The BGS is approximated by a one-period sinusoidal waveform and rectified. Its phase delay, which corresponds to the BFS, is then detected using an XOR logic gate and an LPF. The output voltage is in one-to-one correspondence with the phase delay in the range from 0 to $180^{\circ}$. 
virtual oscilloscope triggered by the repetition frequency for the distributed measurement.

\section{RESULTS AND DISCUSSION}

\section{Operation confirmation}

First, the VCO operation with a BFS sampling rate of $100 \mathrm{kHz}$ was confirmed (an even higher sampling rate was achievable if the SNR deterioration was admitted). When the frequency-control voltage was linearly applied to the VCO (blue line in Figure 3a), the output frequency was distorted (blue curve in Figure $3 \mathrm{~b}$ ) because of the nonlinear frequency dependence on the applied voltage. Therefore, we applied a pre-distorted voltage (red curve in Figure $3 \mathrm{a}$ ) and obtained the linear output frequency (red line in Figure $3 \mathrm{~b}$ ). The output frequency was swept from 2.17 to $2.53 \mathrm{GHz}$, which was set to be sufficiently lower than the BGS $(\sim 10.8 \mathrm{GHz})$ of a silica SMF. If this frequency were close to the BGS, the differential component of their mixed signal would be located near DC and overlapped by its own folded spectrum, resulting in a significantly deteriorated SNR.

Next, we verified that the phase delay and the final DC voltage were in one-to-one correspondence with the applied strain. A 12.8-m-long silica SMF (composed of a 0.8-m-long pigtail of an optical circulator connected to a 12-m-long SMF using an FC/APC adaptor) was used as the FUT. Strains of up to $0.30 \%$ were applied to a $0.4-\mathrm{m}$-long section (6.6-7.0 $\mathrm{m}$ away from the circulator) (Figure 4). The open end was cut at an angle of $8^{\circ}$ in order to suppress the Fresnel reflection. The modulation frequency $f_{\mathrm{m}}$ was set to $7.222 \mathrm{MHz}$, with a correlation peak located at the midpoint of the strained section. The measurement range $d_{\mathrm{m}}$ was calculated to be $14.2 \mathrm{~m}$ according to Equation (1). The modulation amplitude $\Delta f$ was set to $0.35 \mathrm{GHz}$, resulting in a theoretical spatial resolution $\Delta z$ of $\sim 0.38 \mathrm{~m}$ from Equation (2). The ratio of the measurement range to the spatial resolution was $\sim 37$, which can be extended to $\sim 570$ simply by increasing $\Delta f$ to half of the BFS, i.e. $\sim 5.4 \mathrm{GHz}$ (the $\Delta f$ values were kept relatively low to avoid damage to the laser, which was not designed for large-amplitude modulation use). The 64th correlation peak was used, and the room temperature was $18^{\circ} \mathrm{C}$.

The raw and sinusoidally approximated BGSs with and without $0.07 \%$ strain are shown in Figure 5. The vertical axis was normalised so that the maximal and minimal powers of each spectrum became 1 and -1 , respectively. Averaging was performed 10 times. The peaks of the raw BGSs, i.e. BFSs, were not completely the same as those of the approximated BGSs. This is natural considering that the approximation was performed using the whole shape of the BGS, and the strain can still be correctly detected so long as the actual BFS and the peak of

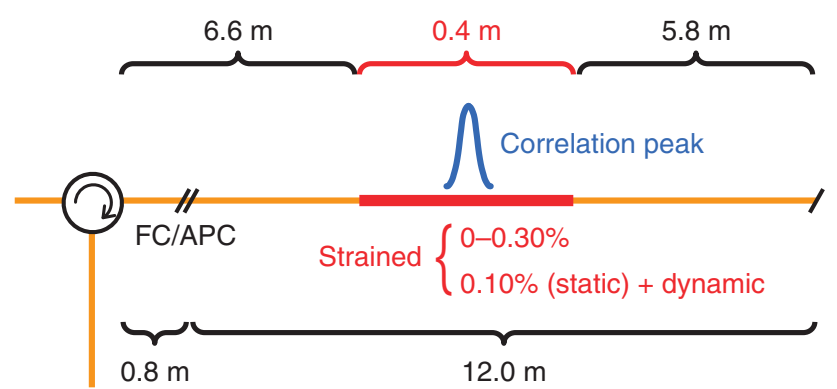

Figure 4 Structures of FUT. Strains of up to $0.30 \%$ were applied to a 0.4-m-long section of a 12.8-m-long silica SMF, which was composed of a 0.8-m-long pigtail of an optical circulator connected to a 12-m-long SMF using an FC/APC adaptor.

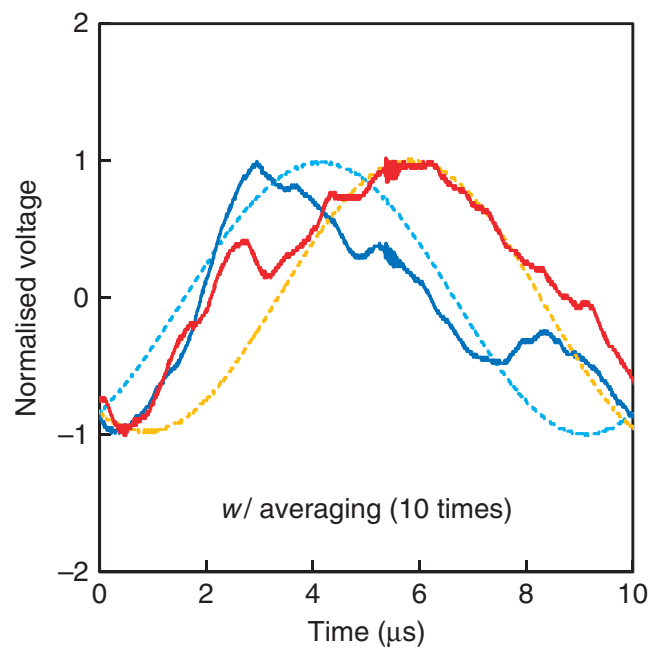

Figure 5 Normalised raw and sinusoidally approximated BGSs. The red and blue solid curves indicate the raw BGSs with and without $0.07 \%$ strain, respectively; whereas the orange and aqua dashed curves are the approximated BGSs with and without $0.07 \%$ strain, respectively. Averaging was performed 10 times.
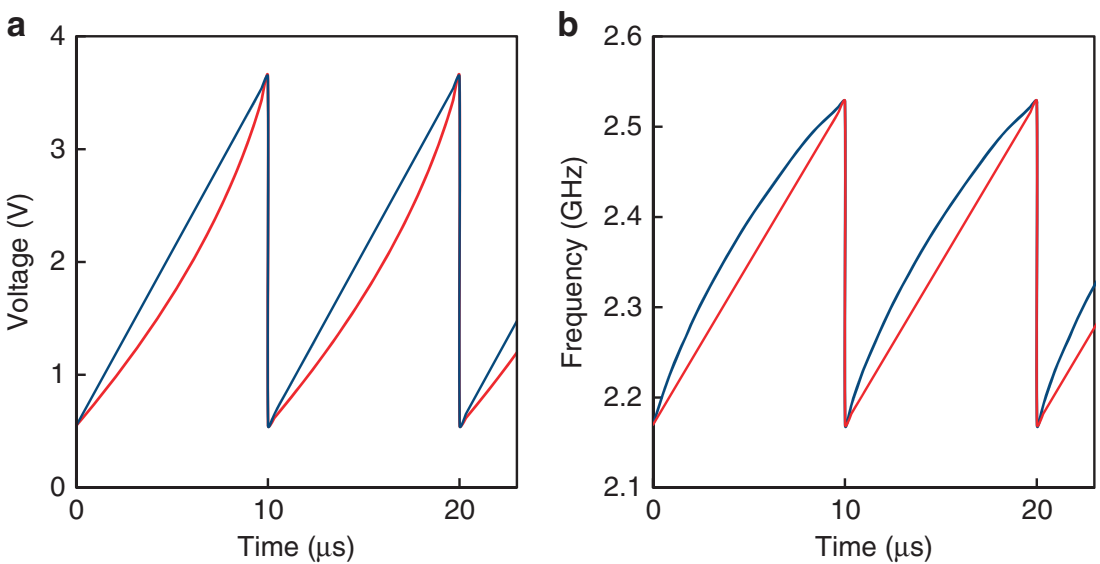

Figure 3 VCO operation with a BFS sampling rate of $100 \mathrm{kHz}$. (a) Linearly applied voltage (blue) and pre-distorted applied voltage (red). (b) Output frequencies when the voltage was applied in linear (blue) and pre-distorted (red) manners. 
the approximated BGS are in one-to-one correspondence (to be verified in the following paragraph). The raw and sinusoidally approximated BGSs with no averaging and 100 times averaging are also shown in Supplementary Fig. S1a and S1b, respectively. As the average number of times increased, the SNR was improved, which indicates that a $100 \mathrm{kHz}$ sampling rate can be obtained if a low SNR is admitted.

The strain dependence of the normalised BGS approximated by a one-period sinusoidal waveform is then shown in Figure 6. Averaging was performed 10 times. The phase was delayed with increasing strain, but the dependence was not completely linear. The phase delay dependence on strain of up to $0.20 \%$ (Figure $7 \mathrm{a}$ ) was almost linear with a slope of 952 degrees/\% (calculated excluding the data at $>0.20 \%$, which deviated from the linear trend). Here, to evaluate the measurement accuracy, we displayed the error bars calculated at each strain as the standard deviations of 100 data plots. The possible error was not constant at different strains and was largest $\left(+/-15.4^{\circ}\right)$ at $\sim 0.15 \%$ strain, because of the spectral noise structure unique to the

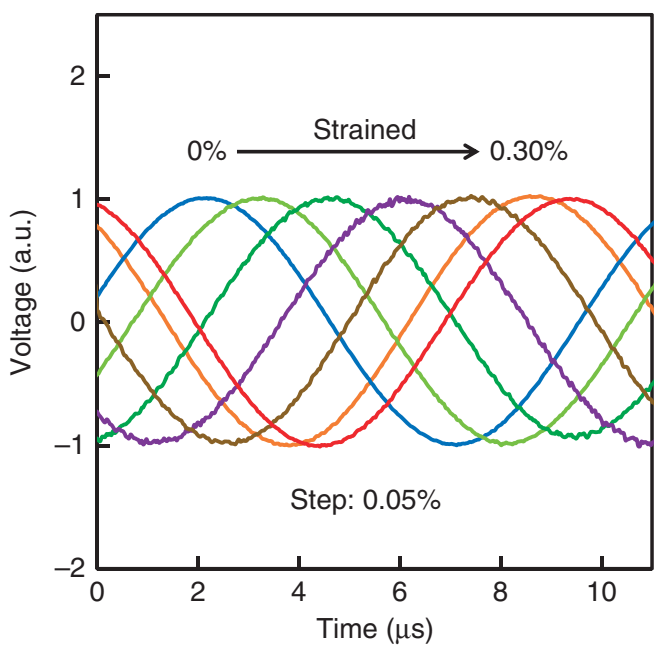

Figure 6 Strain dependence of the normalised BGS approximated by a oneperiod sinusoidal waveform. The spatial resolution $\Delta z$ was $0.38 \mathrm{~m}$, and the length of the strained section $L_{\varepsilon}$ was $0.4 \mathrm{~m}$. Averaging was performed 10 times.
ESA. In the worst case, the measurement error was approximately $+/-10 \%$. We admit that this value is not sufficiently low for accurate strain measurements, but this system is still useful in some relatively rough dynamic strain measurements.

The final DC voltage, i.e. the output from the LPF (cut-off frequency $=1.2 \mathrm{kHz}$ ) was also plotted as a function of applied strain (Figure $7 \mathrm{~b}$ ). The voltage increased linearly with increasing strain (slope $=3.2 \mathrm{~V} / \%$ ) and reached a maximum at $\sim 0.2 \%$, where the phase delay became $180^{\circ}$. At this stage, it decreased for strains beyond $\sim 0.2 \%$. The DC voltage was thus confirmed to correspond to the applied strain, but with a limited strain dynamic range.

Finally, we investigate the error of our phase-based detection, which is possibly induced by the modification of the BGS due to the irregular strain distribution in the vicinity of the correlation peak. In general, the BGS in the correlation-domain techniques consists of two spectral contributions: one is the sharp signal peak corresponding to the strain and the other is the sum of the noise substructure originating from all the other positions ${ }^{23}$. Therefore, the strain distribution at the positions near the correlation peak may modify the overall shape of the BGS, although the peak frequency is maintained. Figure 8 shows one of some extreme cases where large strains are applied near the correlation peak (refer to Supplementary Information for details), and one can see significant modification of the BGS. In order to quantify the possible error of the phase-based detection caused by such a 'neighbourhood effect', we performed some simulations using the method detailed in Ref. 24 with various strain distributions near the correlation peak. As detailed in Supplementary Information, we can conclude that if we allow an absolute error of $+/-200 \mu \varepsilon$ (or $+/-10 \mathrm{MHz}$ ), the spatial resolution is effectively lowered by $\sim 3$ times. It should be also noted that the drop in performance is dependent on the measurement parameters such as the frequency sweep range, FUT length, and maximal applied strain. This finding will be a universal guideline, which is useful not only for this method but also for the other spectral-shape-based methods such as slope-assisted BOCDR ${ }^{37}$.

\section{Demonstrations}

The first demonstration was dynamic strain measurement. A $0.10 \%$ static strain was applied in advance to a 0.4-m-long section (6.6-7.0 m) of the 12.8-m-long silica SMF (Figure 4); the static strain was applied because it was difficult to stably apply dynamic strains to a
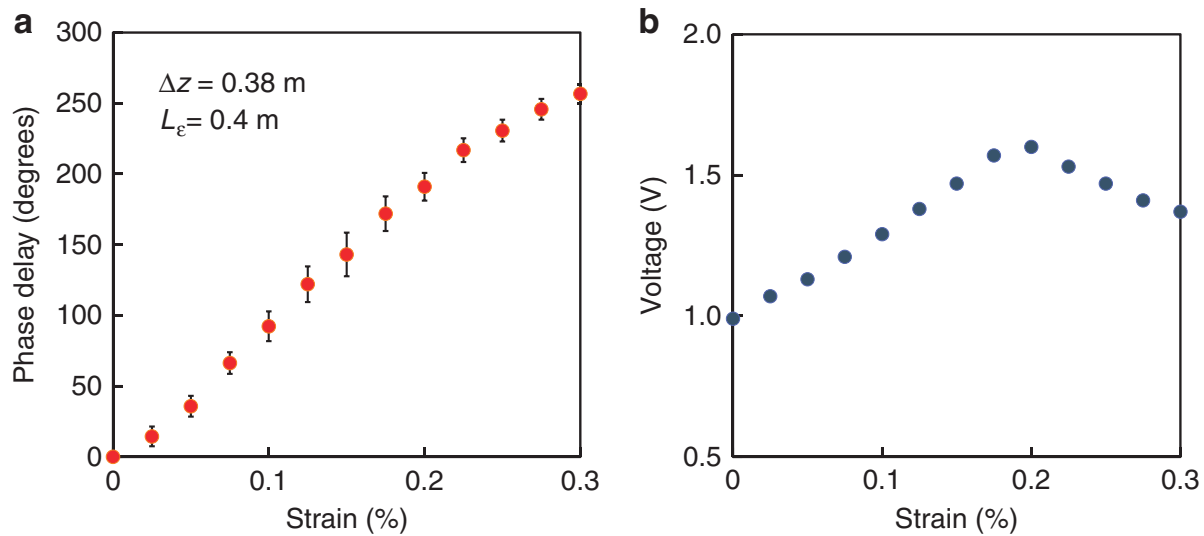

Figure 7 Phase delay and output DC voltage measured as functions of applied strain. (a) Strain dependence of the phase delay. The spatial resolution $\Delta z$ was $0.38 \mathrm{~m}$, and the length of the strained section $L_{\varepsilon}$ was $0.4 \mathrm{~m}$. Averaging was performed 10 times. The error bars were calculated at each strain as the standard deviations of 100 data plots. The slope in the range from 0 to $0.20 \%$ was 952 degrees/\%. (b) Strain dependence of the output DC voltage. The slope in the range from 0 to $0.20 \%$ was $3.2 \mathrm{~V} / \%$. 
non-strained section. Dynamic strains at $30 \mathrm{~Hz}, 100 \mathrm{~Hz}, 300 \mathrm{~Hz}$ and $1 \mathrm{kHz}$ were then applied to the same section using a vibration generator, which makes it unnecessary to consider the neighbourhood effect discussed in the previous section. Averaging was performed 10

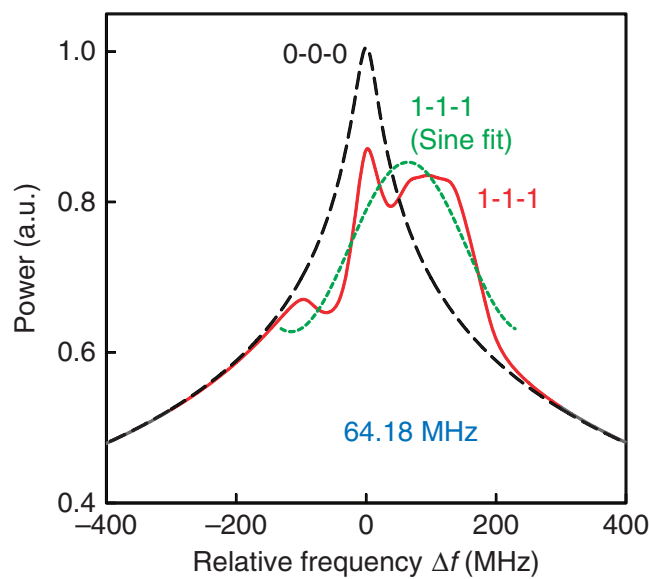

Figure 8 Example of the simulated BGSs. The black dashed curve is the simulated BGS for the FUT configuration '0-0-0' (refer to Supplementary Fig. S2 for the definition), and the red solid curve is that for '1-1-1'. The green dotted curve, which is the sinusoidal fit of the solid curve, becomes maximal at $\Delta f=64.18 \mathrm{MHz}$.
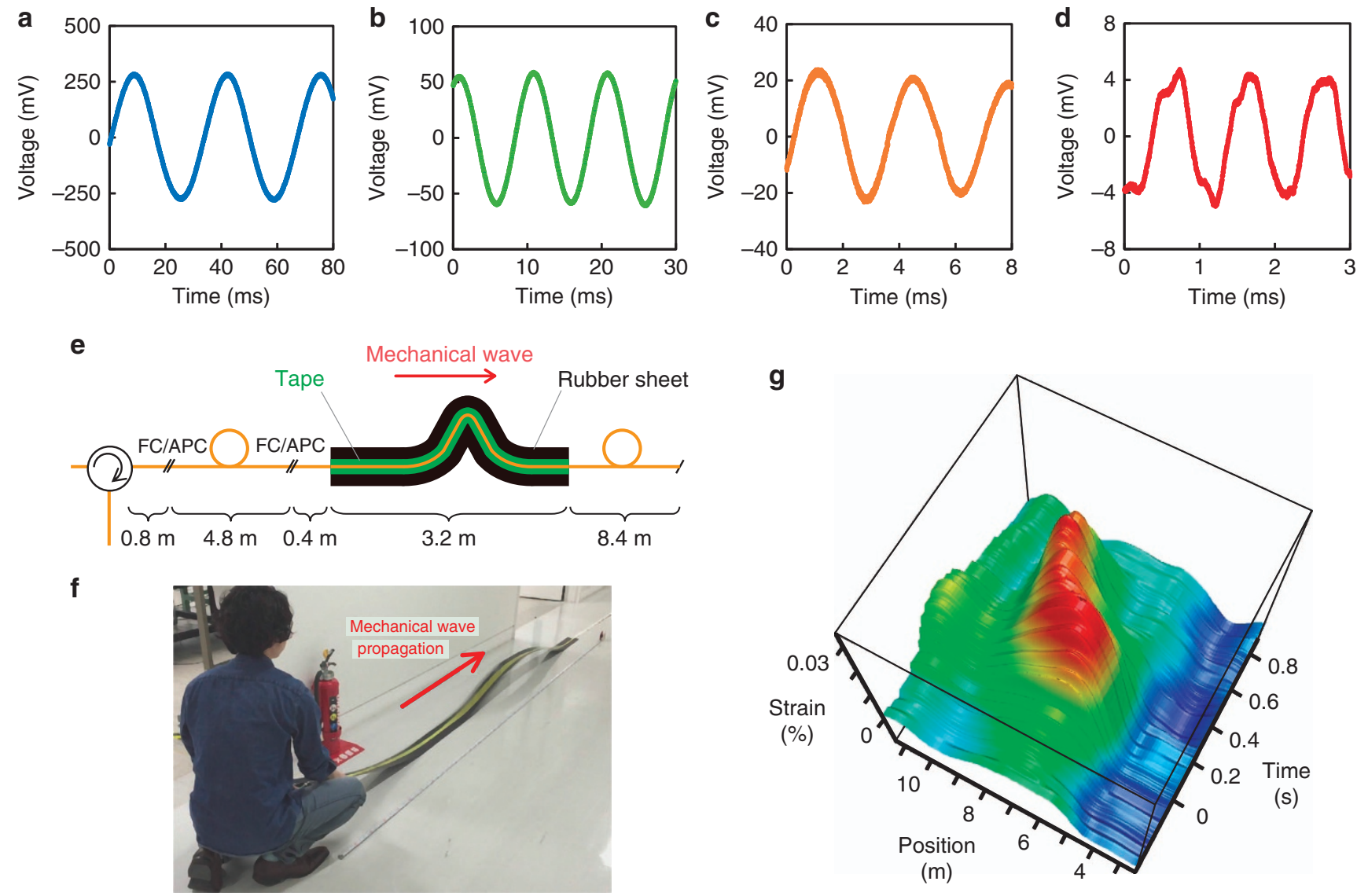

Figure 9 Ultrahigh-speed measurement results. Temporal variations (deviation from the initial value) of the output DC voltage when dynamic strains were applied to a 0.4-m-long section at (a) $30 \mathrm{~Hz}$, (b) $100 \mathrm{~Hz}$, (c) $300 \mathrm{~Hz}$ and (d) $1 \mathrm{kHz}$. (e) Schematic structure and (f) photograph of the FUT while tracking the propagation of a mechanical wave. (g) Measured temporal variation of the strain distribution. 
The measured temporal variation of the strain distribution around the 3.2-m-long section attached to the rubber sheet (Figure 9g) shows that the mechanical wave propagation was detected, though the SNR was low. The propagation speed was calculated to be $\sim 10 \mathrm{~m} \mathrm{~s}^{-1}$. The length of the strained section and the amplitude of the strain decreased as the mechanical wave propagated, which is consistent with the actual observation (Figure 9f). The amount of strain caused by the wave propagation $(<0.03 \%)$ seems to be valid, as it is close to that measured by two-end-access BOTDA ${ }^{39}$. The amount of strain along the 4.8-m-long SMF with a lower BFS was not correctly detected (negative values obtained), which is reasonable considering the operating principle that limits the measurable strain range. The negative-strain (i.e. compressed) regions, which should appear at the foot of the peak ${ }^{39}$ were not clearly detected probably because of the combination of the following three reasons: (i) the dependence coefficient of the phase delay on negative strain (compression) was smaller, as the experimental setup was optimised for strain ranging from 0 to $\sim 0.2 \%$; (ii) the low-pass filtering at the end of the signal processing reduced the drop of the voltage; (iii) the properties of the materials (rubber, tape and so on) used to fabricate the FUT were different from those in Ref. 39. In this way, although the SNR was not sufficiently high, the ultrahigh-speed distributed strain sensing capability of this system was demonstrated.

\section{CONCLUSIONS}

In this work, real-time distributed Brillouin reflectometry with intrinsic one-end accessibility and high spatial resolution was developed for the first time by use of a correlation-domain technique. In this configuration, the BGS was converted from frequency domain to time domain by mixing it with the output of a VCO. Then, the BGS in the time domain was approximated by a one-period sinusoidal waveform, and the BFS was converted into its phase delay, which was subsequently converted into a voltage so that the BFS information can be directly obtained. A strain sampling rate of up to $100 \mathrm{kHz}$ at an arbitrary position was experimentally verified by detecting locally applied dynamic strain at $1 \mathrm{kHz}$. A repetition rate of distributed measurement of $100 \mathrm{~Hz}$ (10 times averaging, 100 sensing points) was also verified by tracking a propagating mechanical wave. We must admit that, compared with those of other high-speed techniques, including slope-assisted BOTDA ${ }^{5,6}$ and BOCDA ${ }^{19,20,25}$, the current performance of our method is low because of its major drawbacks, such as the relatively low measurement accuracy (in the worst case, approximately $+/-10 \%$ error with 10 times averaging), spatial resolution degraded by a factor of $\sim 3$ (compared with standard $\mathrm{BOCDR}^{30,31}$ or $\mathrm{BOCDA}^{17}$ ), and limited strain dynamic range (0 to $\sim 0.2 \%$ in this experiment); these points could be a margin for improvement in the future. However, we anticipate that intrinsic oneend accessibility, the advantage of this system that other techniques cannot provide, will more than compensate for these shortcomings and that this will be a promising technique for distributed dynamic strain and temperature sensing with practical convenience, especially for a relatively short measurement range.

\section{CONFLICT OF INTEREST}

The author declare no conflict of interest.

\section{AUTHOR CONTRIBUTIONS}

YM and KN designed the study. YM and NH performed the experiments. YM and HF analysed the data. YM and KYS performed the simulation. YM wrote the manuscript with input from all co-authors.

\section{ACKNOWLEDGEMENTS}

We wish to acknowledge Tomohito Kawa, Heeyoung Lee, Shumpei Shimada, Makoto Shizuka, Kazunari Minakawa, Hiroki Tanaka, Wei Qiu, Sho Ikeda, Daisuke Yamane, Hiroyuki Ito, Shiro Dosho and Kazuya Masu (Institute of Innovative Research, Tokyo Institute of Technology) for their experimental assistance and Richard Nedelcov (Department of Language Arts, Tokyo University of the Arts) for his English editing. This work was supported by JSPS KAKENHI Grant Numbers 25709032, 26630180 and 25007652, and by research grants from the Iwatani Naoji Foundation, the SCAT Foundation and the Konica Minolta Science and Technology Foundation.

1 Agrawal GP. Nonlinear Fibre Optics. New York: Academic Press; 2001.

2 Horiguchi T, Tateda M. BOTDA —nondestructive measurement of single-mode optical fibre attenuation characteristics using Brillouin interaction: theory. J Lightwave Technol 1989; 7: 1170-1176.

3 Voskoboinik A, Wang J, Shamee B, Nuccio SR, Zhang L et al. SBS-based fibre optical sensing using frequency-domain simultaneous tone interrogation. J Lightwave Technol 2011; 29: 1729-1735.

4 Bernini R, Minardo A, Zeni L. Dynamic strain measurement in optical fibres by stimulated Brillouin scattering. Opt Lett 2009; 34: 2613-2615.

5 Peled Y, Motil A, Yaron L, Tur M. Slope-assisted fast distributed sensing in optical fibres with arbitrary Brillouin profile. Opt Express 2011; 19: 19845-19854.

6 Peled Y, Motil A, Tur M. Fast Brillouin optical time domain analysis for dynamic sensing. Opt Express 2012; 20: 8584-8591.

7 Taki M, Muanenda Y, Oton CJ, Nannipieri T, Signorini A et al. Cyclic pulse coding for fast BOTDA fibre sensors. Opt Lett 2013; 38: 2877-2880.

8 Danon 0, Motil A, Sovran I, Hadar R, Tur M. Real-time fast and distributed measurement of a Brillouin-inhomogeneous fibre using tailored-frequency probe in slope-assisted BOTDA. Proc SPIE 2014; 9157: 9157AM.

9 Muanenda Y, Taki M, Pasquale FD. Long-range accelerated BOTDA sensor using adaptive linear prediction and cyclic coding. Opt Lett 2014; 39: 5411-5414.

10 Sovran I, Motil A, Tur M. Frequency-scanning BOTDA with ultimately fast acquisition speed. IEEE Photonics Technol Lett 2015; 27: 1426-1429.

11 Dong YK, Ba DX, Jiang TF, Zhou DW, Zhang HY et al. High-spatial-resolution fast BOTDA for dynamic strain measurement based on differential double-pulse and secondorder sideband of modulation. IEEE Photonics J 2013; 5: 2600407.

12 Elooz D, Antman Y, Levanon N, Zadok A. High-resolution long-reach distributed Brillouin sensing based on combined time-domain and correlation-domain analysis. Opt Express 2014; 22: 6453-6463.

13 Garus D, Krebber K, Schliep F, Gogolla T. Distributed sensing technique based on Brillouin optical-fibre frequency-domain analysis. Opt Lett 1996; 21: 1402-1404.

14 Bernini R, Minardo A, Zeni L. Distributed sensing at centimeter-scale spatial resolution by BOFDA: measurements and signal processing. IEEE Photonics J 2012; 4: 48-56.

15 Minardo A, Bernini R, Zeni L. Distributed temperature sensing in polymer optical fibre by BOFDA. IEEE Photonics Technol Lett 2014; 26: 387-390.

16 Wosniok A, Mizuno Y, Krebber K, Nakamura K. L-BOFDA: a new sensor technique for distributed Brillouin sensing. Proc SPIE 2013; 8794: 879431.

17 Hotate K, Hasegawa T. Measurement of Brillouin gain spectrum distribution along an optical fibre using a correlation-based technique — proposal, experiment and simulation. IEICE Trans Electron 2000; E83-C: 405-412.

18 Song KY, He ZY, Hotate K. Distributed strain measurement with millimeter-order spatial resolution based on Brillouin optical correlation domain analysis. Opt Lett 2006; 31: 2526-2528.

19 Song KY, Hotate K. Distributed fibre strain sensor with 1-kHz sampling rate based on Brillouin optical correlation domain analysis. IEEE Photonics Technol Lett 2007; 19: 1928-1930.

20 Song KY, Kishi M, He ZY, Hotate K. High-repetition-rate distributed Brillouin sensor based on optical correlation-domain analysis with differential frequency modulation. Opt Lett 2011; 36: 2062-2064.

21 Song KY, Hotate K. Brillouin optical correlation domain analysis in linear configuration. IEEE Photonics Technol Lett 2008; 20: 2150-2152.

22 Jeong JH, Chung KH, Lee SB, Song KY, Jeong JM et al. Linearly configured BOCDA system using a differential measurement scheme. Opt Express 2014; 22 1467-1473.

23 Song KY, He ZY, Hotate K. Optimization of Brillouin optical correlation domain analysis system based on intensity modulation scheme. Opt Express 2006; 14: 4256-4263.

24 Song KY, He ZY, Hotate K. Effects of intensity modulation of light source on Brillouin optical correlation domain analysis. J Lightwave Technol 2007; 25: 1238-1246.

25 Zhang CY, Kishi M, Hotate K. 5,000 points/s high-speed random accessibility for dynamic strain measurement at arbitrary multiple points along a fibre by Brillouin optical correlation domain analysis. Appl Phys Express 2015; 8: 042501.

26 Kurashima T, Horiguchi T, Izumita H, Furukawa S, Koyamada Y. Brillouin optical-fibre time domain reflectometry. IEICE Trans Commun 1993; E76-B: 382-390.

27 Alahbabi MN, Cho YT, Newson TP. 100 km distributed temperature sensor based on coherent detection of spontaneous Brillouin backscatter. Meas Sci Technol 2004; 15: 1544-1547. 
28 Geng J, Staines S, Blake M, Jiang SB. Distributed fibre temperature and strain sensor using coherent radio-frequency detection of spontaneous Brillouin scattering. Appl Opt 2007; 46: 5928-5932.

29 Masoudi A, Belal M, Newson TP. Distributed dynamic large strain optical fibre sensor based on the detection of spontaneous Brillouin scattering. Opt Lett 2013; 38: 3312-3315.

30 Tu GJ, Zhang XP, Zhang YX, Ying ZF, Lv LD. Strain variation measurement with shorttime Fourier transform-based Brillouin optical time-domain reflectometry sensing system. Electron Lett 2014; 50: 1624-1626.

31 Mizuno Y, Zou WW, He ZY, Hotate K. Proposal of Brillouin optical correlation-domain reflectometry (BOCDR). Opt Express 2008; 16: 12148-12153.

32 Mizuno Y, Zou WW, He ZY, Hotate K. Operation of Brillouin optical correlation-domain reflectometry: theoretical analysis and experimental validation. J Lightwave Technol 2010; 28: 3300-3306.

33 Mizuno Y, He ZY, Hotate K. One-end-access high-speed distributed strain measurement with 13-mm spatial resolution based on Brillouin optical correlation-domain reflectometry. IEEE Photonics Technol Lett 2009; 21: 474-476.

34 Mizuno Y, He ZY, Hotate K. Measurement range enlargement in Brillouin optical correlation-domain reflectometry based on temporal gating scheme. Opt Express 2009; 17: 9040-9046.

35 Mizuno Y, He ZY, Hotate K. Stable entire-length measurement of fibre strain distribution by Brillouin optical correlation-domain reflectometry with polarization scrambling and noise-floor compensation. Appl Phys Express 2009; 2: 062403.
36 Manotham S, Kishi M, He ZY, Hotate K. 1-cm spatial resolution with large dynamic range in strain distributed sensing by Brillouin optical correlation domain reflectometry based on intensity modulation. Proc SPIE 2012; 8351: 835136.

37 Lee HY, Hayashi N, Mizuno Y, Nakamura K. Slope-assisted Brillouin optical correlation-domain reflectometry: proof of concept. IEEE Photonics J 2016; 8: 6802807.

38 Hotate K, He ZY. Synthesis of optical-coherence function and its applications in distributed and multiplexed optical sensing. J Lightwave Technol 2006; 24: 2541-2557.

39 Peled Y, Motil A, Kressel I, Tur M. Monitoring the propagation of mechanical waves using an optical fibre distributed and dynamic strain sensor based on BOTDA. Opt Express 2013; 21: 10697-10705.

(c) (i) (2) This work is licensed under a Creative Commons AttributionBY NC SA NonCommercial-ShareAlike 4.0 International License. The images or
other third party material in this article are included in the article's Creative Commons license, unless indicated otherwise in the credit line; if the material is not included under the Creative Commons license, users will need to obtain permission from the license holder to reproduce the material. To view a copy of this license, visit http:// creativecommons.org/licenses/by-nc-sa/4.0/

(C) The Author(s) 2016

Supplementary Information for this article can be found on the Light: Science \& Applications' website (http://www.nature.com/lsa). 